\title{
The first data on the freshwater microcrustaceans of Shokalsky Island (Russian Arctic)
}

\author{
Anna Novichkova $\ddagger$, \\ ‡ Lomonosov Moscow State University, Moscow, Russia \\ § A.N. Severtsov Institute of Ecology and Evolution, Moscow, Russia
}

Corresponding author: Anna Novichkova (anna.hydro@gmail.com)

Academic editor: Niamh Kilgallen

Received: 26 Oct 2016 | Accepted: 22 Dec 2016 | Published: 23 Dec 2016

Citation: Novichkova A (2016) The first data on the freshwater microcrustaceans of Shokalsky Island (Russian

Arctic). Biodiversity Data Journal 4: e10930. https://doi.org/10.3897/BDJ.4.e10930

\section{Abstract}

\section{Background}

Information on freshwater invertebrates of the Russian Arctic is very scarce, especially concerning insular biota. The species composition of microcrustaceans (Cladocera, Copepoda) of many arctic islands is still unknown and have never been explored. Here we report the results of the first investigation of the zooplankton of the Shokalsky Island (YamaloNenets Autonomous Okrug, Russia).

Information on freshwater invertebrates of the Russian Arctic is very scarce, especially concerning insular biota. The species composition of microcrustaceans (Cladocera, Copepoda) of many arctic islands is still unknown and have never been explored. Here we report the results of the first investigation of the zooplankton of the Shokalsky Island (YamaloNenets Autonomous Okrug, Russia).

\section{New information}

The new records reported here are novel for the region and significantly expand the knowledge of the high-latitude aquatic biota. We studied the species composition of 
Cladocera and Copepoda of 21 freshwater habitats located on the southwestern part of Shokalsky Island. We found 15 species of microcrustaceans in total and all of them are reported for the first time here. Also, the obtained data expand the existing ranges of distribution of some species and report several new taxa for the whole YamaloNenets region of Russia.

\section{Keywords}

Cladocera, Copepoda, microcrustaceans, freshwater, Russian Arctic, Shokalsky Island

\section{Introduction}

Shokalsky Island is a small island in the Kara Sea located in the YamaloNenets Autonomous Okrug of Russia (Fig. 1). It is separated from the mainland by a narrow strait, which is only 59 metres in width. The island is a flat plain covered by tundra with a great number of rivers, small lakes and ponds (Il'yna et al. 1985). It belongs to the Gydan Nature Reserve, which includes a diverse terrain of northern Gydan Peninsula, preserving waterfowl nesting areas, polar bear, and walrus and is known as the nothermost nature reserve in Western Siberia (Nikiforov 1997). Due to its remotness and very limited access to some territories, the level of scientifical researches here is rather low and some of the aspects are under studied. Despite the long period of freshwater observations in the region, invertebrate fauna of all the peninsulas lying in the YamaloNenets Autonomous Okrug is known only fragmentarily (Sharapova and Abdullina 2004). According to the latest data, information on the zooplankton of the northen Yamal is very scarce, while the species inhabiting water bodies of Tazovsky and Gydan Peninsulas are known only from rare publications focused on large lakes and rivers (Popov 2012). The species composition of the microcrustaceans (Cladocera, Copepoda) of Shokalsky island has never been explored.

\section{Materials and methods}

The samples were collected during a hydrobiological survey of the compex expedition of KUBZ (Moscow Zoo young biologist's coterie) in August, 2014. Microcrustaceans were collected from 21 freshwater habitats from southwestern part of Shokalsky Island, most of them were small thermokarst ponds with the depth of $0.5-1.5 \mathrm{~m}$ (Fig. 2). Environmental variables such as bottom sediment type (clay, silt, sand, detritus or thick mosses, measured in accordance with Wentworth Grade Scale (Ongley 1996)), average depth (mean value for the whole sampled area) and size (average length of diameters) of the water body were noted for each site (Table 1). The sampling was performed from the shore using a qualitative plankton net (type "Apstein", mesh size $50 \mu \mathrm{m}$ ). Upon collection, all samples were preserved in ethanol (96\%). Species identification and enumeration was carried out primarily in Bogorov counting chambers; the total numbers of Cladocera and 
Copepoda were recorded. Description of the distributional ranges of the species is also provided in the checklist: AT - Afrotropical, AU - Australasian, ANT - Antarctic, NA Nearctic, NT - Neotropical, OL - Oriental, PA - Palaearctic, PAC - Pacific oceanic islands.

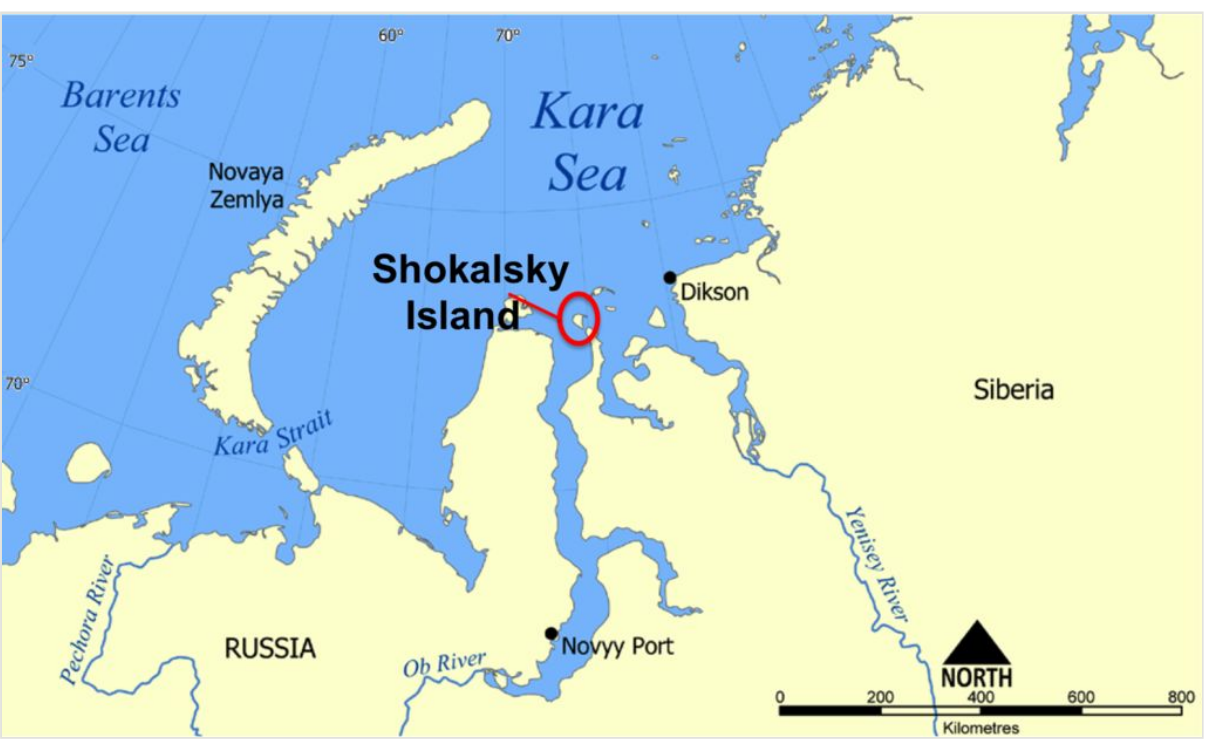

Figure 1.

Location of the Shokalsky Island on the map. Original map from: commons.wikimedia.org.

Table 1.

Locations and dates of sampling with notes on the water body type and substratum (bottom sediment).

\begin{tabular}{|c|c|c|c|c|c|c|c|}
\hline $\begin{array}{l}\text { Site } \\
\text { No. }\end{array}$ & Date & Coordinates & Altitude & $\begin{array}{l}\text { Water body } \\
\text { type }\end{array}$ & $\begin{array}{c}\text { Bottom } \\
\text { Sediment }\end{array}$ & $\begin{array}{c}\text { Average } \\
\text { Size }\end{array}$ & $\begin{array}{c}\text { Average } \\
\text { Depth }\end{array}$ \\
\hline 1 & 03.08 .2014 & $\begin{array}{l}72.91667^{\circ} \mathrm{N} \\
74.33418^{\circ} \mathrm{E}\end{array}$ & $7 \mathrm{~m}$ & bayou pond & silt, mosses & $5 \mathrm{~m} \times 13 \mathrm{~m}$ & $0.5 \mathrm{~m}$ \\
\hline 2 & 03.08.2014 & $\begin{array}{l}72.91928^{\circ} \mathrm{N} \\
74.33338^{\circ} \mathrm{E}\end{array}$ & $2 m$ & bayou pond & silt & $5 \mathrm{~m} \times 10 \mathrm{~m}$ & $0.5 \mathrm{~m}$ \\
\hline 3 & 03.08 .2014 & $\begin{array}{l}72.92120^{\circ} \mathrm{N} \\
74.33328^{\circ} \mathrm{E}\end{array}$ & $-6 m$ & bayou pond & silt & $8 \mathrm{~m} \times 20 \mathrm{~m}$ & $0.3 \mathrm{~m}$ \\
\hline 4 & 04.08.2014 & $\begin{array}{l}72.91103^{\circ} \mathrm{N} \\
74.39402^{\circ} \mathrm{E}\end{array}$ & $-1 m$ & $\begin{array}{l}\text { thermokarst } \\
\text { pond }\end{array}$ & silt, detritus & $6 \mathrm{~m} \times 10 \mathrm{~m}$ & $0.5 \mathrm{~m}$ \\
\hline 5 & 04.08 .2014 & $\begin{array}{l}72.91523^{\circ} \mathrm{N} \\
74.40652^{\circ} \mathrm{E}\end{array}$ & $7 \mathrm{~m}$ & $\begin{array}{l}\text { thermokarst } \\
\text { pond }\end{array}$ & clay, sand & $15 \mathrm{~m} \times 30 \mathrm{~m}$ & $1.5 \mathrm{~m}$ \\
\hline
\end{tabular}




\begin{tabular}{|c|c|c|c|c|c|c|c|}
\hline 6 & 05.08.2014 & $\begin{array}{l}72.94028^{\circ} \mathrm{N} \\
74.33675^{\circ} \mathrm{E}\end{array}$ & $3 m$ & $\begin{array}{l}\text { thermokarst } \\
\text { pond }\end{array}$ & mosses & $1.5 \mathrm{~m} \times 15 \mathrm{~m}$ & $1.5 \mathrm{~m}$ \\
\hline 7 & 05.08.2014 & $\begin{array}{l}72.94101^{\circ} \mathrm{N} \\
74.33377^{\circ} \mathrm{E}\end{array}$ & & boggy stream & mosses & $1 \mathrm{~m} \times 1.5 \mathrm{~m}$ & $1.5 \mathrm{~m}$ \\
\hline 8 & 06.08.2014 & $\begin{array}{l}72.96975^{\circ} \mathrm{N} \\
74.33328^{\circ} \mathrm{E}\end{array}$ & $-1 m$ & $\begin{array}{l}\text { thermokarst } \\
\text { pond }\end{array}$ & clay, silt & $5 \mathrm{~m} \times 10 \mathrm{~m}$ & $0.5 \mathrm{~m}$ \\
\hline 9 & 07.08 .2014 & $\begin{array}{l}72.93123^{\circ} \mathrm{N} \\
74.29818^{\circ} \mathrm{E}\end{array}$ & $3 m$ & bayou pond & sand, mosses & $3 m \times 5 m$ & $0.5 \mathrm{~m}$ \\
\hline 10 & 07.08.2014 & $\begin{array}{l}72.93850^{\circ} \mathrm{N} \\
74.30105^{\circ} \mathrm{E}\end{array}$ & $0 \mathrm{~m}$ & $\begin{array}{l}\text { thermokarst } \\
\text { pond }\end{array}$ & sand, mosses & $2 m \times 5 m$ & $1.5 \mathrm{~m}$ \\
\hline 11 & 10.08 .2014 & $\begin{array}{l}72.93318^{\circ} \mathrm{N} \\
74.40840^{\circ} \mathrm{E}\end{array}$ & $-1 m$ & $\begin{array}{l}\text { thermokarst } \\
\text { pond }\end{array}$ & silt, mosses & $5 \mathrm{~m} \times 10 \mathrm{~m}$ & $1 \mathrm{~m}$ \\
\hline 12 & 10.08.2014 & $\begin{array}{l}72.93198^{\circ} \mathrm{N} \\
74.29845^{\circ} \mathrm{E}\end{array}$ & $8 m$ & $\begin{array}{l}\text { thermokarst } \\
\text { pond }\end{array}$ & sand, mosses & $1.5 \mathrm{~m} \times 6 \mathrm{~m}$ & $0.5 \mathrm{~m}$ \\
\hline 13 & 11.08 .2014 & $\begin{array}{l}72.93358^{\circ} \mathrm{N} \\
74.29932^{\circ} \mathrm{E}\end{array}$ & $3 m$ & $\begin{array}{l}\text { thermokarst } \\
\text { pond }\end{array}$ & mosses & $2 \mathrm{~m} \times 2.5 \mathrm{~m}$ & $1 \mathrm{~m}$ \\
\hline 14 & 12.08.2014 & $\begin{array}{l}72.95233^{\circ} \mathrm{N} \\
74.34007^{\circ} \mathrm{E}\end{array}$ & $3 m$ & $\begin{array}{l}\text { thermokarst } \\
\text { pond }\end{array}$ & sand, mosses & $2 m \times 10 m$ & $0.5 \mathrm{~m}$ \\
\hline 15 & 12.08 .2014 & $\begin{array}{l}72.94750^{\circ} \mathrm{N} \\
74.33728^{\circ} \mathrm{E}\end{array}$ & $9 m$ & $\begin{array}{l}\text { thermokarst } \\
\text { pond }\end{array}$ & silt, mosses & $1.5 \mathrm{~m} \times 2 \mathrm{~m}$ & $1.5 \mathrm{~m}$ \\
\hline 16 & 13.08.2014 & $\begin{array}{l}72.93787^{\circ} \mathrm{N} \\
74.39213^{\circ} \mathrm{E}\end{array}$ & $1 \mathrm{~m}$ & $\begin{array}{l}\text { thermokarst } \\
\text { pond }\end{array}$ & silt, mosses & $5 \mathrm{~m} \times 20 \mathrm{~m}$ & $2 m$ \\
\hline 17 & 13.08.2014 & $\begin{array}{l}72.95078^{\circ} \mathrm{N} \\
74.38572^{\circ} \mathrm{E}\end{array}$ & Om & $\begin{array}{l}\text { thermokarst } \\
\text { pond }\end{array}$ & sand, silt & $10 \mathrm{~m} \times 30 \mathrm{~m}$ & $2 m$ \\
\hline 18 & 14.08 .2014 & $\begin{array}{l}72.93840^{\circ} \mathrm{N} \\
74.42920^{\circ} \mathrm{E}\end{array}$ & & $\begin{array}{l}\text { thermokarst } \\
\text { pond }\end{array}$ & silt, mosses & $5 \mathrm{~m} \times 20 \mathrm{~m}$ & $1 \mathrm{~m}$ \\
\hline 19 & 15.08 .2014 & $\begin{array}{l}72.93803^{\circ} \mathrm{N} \\
74.34598^{\circ} \mathrm{E}\end{array}$ & $-9 m$ & $\begin{array}{l}\text { thermokarst } \\
\text { pond }\end{array}$ & $\begin{array}{l}\text { silty sand, } \\
\text { mosses }\end{array}$ & $20 \mathrm{~m} \times 40 \mathrm{~m}$ & $1 \mathrm{~m}$ \\
\hline 20 & 15.08 .2014 & $\begin{array}{l}72.94862^{\circ} \mathrm{N} \\
74.46823^{\circ} \mathrm{E}\end{array}$ & $0 \mathrm{~m}$ & $\begin{array}{l}\text { thermokarst } \\
\text { pond }\end{array}$ & clay & $20 m \times 45 m$ & $1.5 \mathrm{~m}$ \\
\hline 21 & 15.08 .2014 & $\begin{array}{l}72.98183^{\circ} \mathrm{N} \\
74.51590^{\circ} \mathrm{E}\end{array}$ & $7 m$ & lake & sand & $\begin{array}{l}450 m x \\
550 m\end{array}$ & $1.5 \mathrm{~m}$ \\
\hline
\end{tabular}




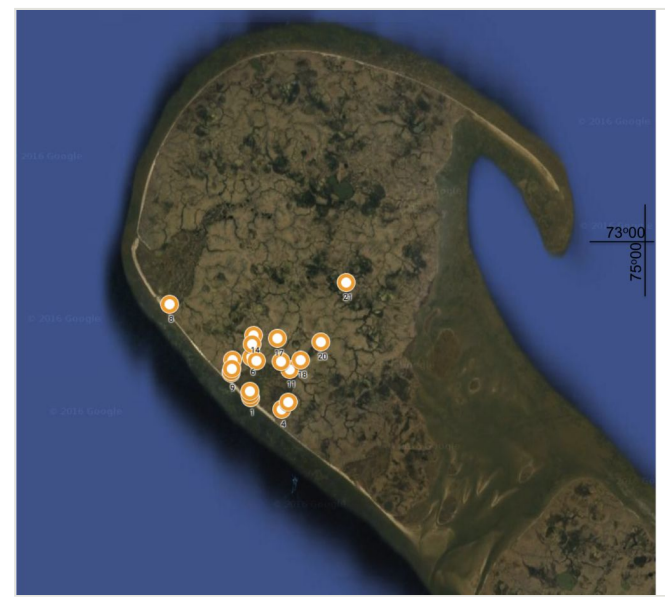

Figure 2.

Location of the sampled stations in inner water bodies on the map of Shokalsky Island (Google Maps).

\section{List of species Cladocera and Copepoda recorded on Shokalsky} Island

Infraorder Cladocera Latreille, 1829

Family Chydoridae Dybowski et Grochowski, 1894

Acroperus harpae (Baird, 1834)

Notes: localities no. 1, 7. Distribution: AT, AU, NA, NT, OL, PA.

Alona affinis (O.F. Müller, 1776)

Notes: localities no. 1, 3, 5, 6, 7, 16, 21. Distribution: AT, AU, NA, NT, OL, PA.

Alona quadrangularis (Leydig, 1860)

Notes: locality no. 9. Distribution: AT, AU, NA, OL, PA.

Chydorus sphaericus (O.F. Müller, 1776)

Notes: localities no. 1-9, 11, 13, 16, 19, 20, 21. Distribution: AT, AU, NA, NT, OL, PAC, PA. 
Graptoleberis testudinaria (Fischer, 1848)

Notes: locality no. 17. Distribution (subsp. testudinaria): AT, AU, NA, NT, OL, PA.

Family Eurycercidae Kurz, 1875 sensu Dumont et Silva-Briano, 1998

Eurycercus (Teretifrons) glacialis Lilljeborg 1887

Notes: localities no. 1, 21. Distribution: NA, PA.

Family Daphniidae Straus, 1820

Daphnia cf. pulex Leydig, 1860

Notes: localities no. 3, 5, 6, 7, 13, 19. Distribution: AT, NA, NT, PA.

Scapholeberis mucronata (O.F. Müller, 1776)

Notes: localities no. 1, 6, 7. Distribution: NA, NT, PA.

Simocephalus vetulus (O.F. Müller, 1776)

Notes: locality no. 1. Distribution: PA.

Family Bosminidae Baird 1845 sensu Sars 1865

Bosmina (Bosmina) longirostris (O. F. Müller, 1785)

Notes: localities no. 17, 18. Distribution: : AT, ANT, AU, NA, NT, OL, PAC, PA.

Family Sididae Baird, 1850

Latona setifera (O.F. Müller, 1776)

Notes: localities no. 21. Distribution: NA, PA.

Family Polyphemidae Baird, 1845

Polyphemus pediculus (Linnaeus, 1761)

Notes: localities no. 1-9, 13, 14, 20, 21. Distribution: NA, PA 
Subclass Copepoda Milne Edwards, 1840

Order Cyclopoida Burmeister, 1834

Family Cyclopidae Rafinesque, 1815

Cyclops vicinus Uljanin, 1875

Notes: localities no. 1, 4, 18, 19.

Order Calanoida Sars G.O., 1903

Family Diaptomidae Baird, 1850

Diaptomus cf. castor (Jurine, 1820)

Notes: locality no. 1. Distribution: PA (Europe (Austria, France....), Greenland, Northern Alaska (Colville River)).

Leptodiaptomus angustilobius (Sars G.0., 1898)

Notes: localities no. 1-9, 12, 13, 14, 18, 20, 21. Distribution: NA (Arctic and Subarctic Canada, to the Kuril Islands).

\section{Discussion}

In total 15 species of microcrustaceans were identified, comprising 12 species in 12 genera of Cladocera, and three species in three genera of Copepoda. All of the taxa have not been previously documented on the island. Microcrustaceans were found in $90 \%$ of the studied sites. The number of species encountered in each water body varied from one to ten (Table 2). The most common species in the studied sites were Leptodiaptomus angustilobius (Sars, 1898), Polyphemus pediculus (Linnaeus, 1761) and Chydorus cf. sphaericus (Muller, 1776), they usually dominate in the communities and occured in most of the investigated water bodies.

The distributional ranges of all the species are rather wide, none of them are restricted to the arctic area or more limited region. The areas of the species are noted in the Checklist according to the FADA Databases of Cladocera (Kotov et al. 2013) and Copepoda (Boxshall and Defaye 2009). The most important findings are Latona setifera (Muller, 1776), Diaptomus cf. castor (Jurine, 1820) and Graptoleberis testudinaria (Fischer, 1848). The first two species have never been found on the territory of Yamalo-Nenets Autonomous Okrug, and the third one was only known from waters of lower Ob' River (Semyonova et al. 
2000). All of them occured rarely in separate water bodies. For the species $L$. setifera this record is the northernmost finding ever (Korovchinsky 2004).

Table 2.

Main characteristics of microcrustacean communities in the observed water bodies.

\begin{tabular}{|c|c|c|c|}
\hline Site No. & $\begin{array}{l}\text { Dominant species } \\
\text { (\% of total abundance) }\end{array}$ & $\begin{array}{l}\text { Subdominants } \\
\text { (\% of total abundance) }\end{array}$ & $\begin{array}{c}\text { Total number of } \\
\text { species }\end{array}$ \\
\hline 1 & $\begin{array}{l}\text { Scapholeberis mucronata }(20 \%)+ \\
\text { Chydorus sphaericus }(19,2 \%)\end{array}$ & Simocephalus vetulus $(15,8 \%)$ & 10 \\
\hline 2 & Leptodiaptomus angustilobius $(83,3 \%)$ & Polyphemus pediculus (12,5\%) & 3 \\
\hline 3 & Polyphemus pediculus $(90,8 \%)$ & Chydorus sphaericus (6,5\%) & 5 \\
\hline 4 & Leptodiaptomus angustilobius $(99,7 \%)$ & - & 4 \\
\hline 5 & Leptodiaptomus angustilobius (97,9\%) & - & 5 \\
\hline 6 & Leptodiaptomus angustilobius $(70,4 \%)$ & Chydorus sphaericus $(23,5 \%)$ & 6 \\
\hline 7 & Polyphemus pediculus $(62,1 \%)$ & Chydorus sphaericus $(15,3 \%)$ & 7 \\
\hline 8 & Polyphemus pediculus $(57,1 \%)$ & $\begin{array}{l}\text { Chydorus sphaericus }(28,6 \%)+ \\
\text { Leptodiaptomus angustilobius (14,3\%) }\end{array}$ & 3 \\
\hline 9 & Chydorus sphaericus (51\%) & Polyphemus pediculus (36\%) & 4 \\
\hline 10 & - & - & 0 \\
\hline 11 & Chydorus sphaericus (100\%) & - & 1 \\
\hline 12 & Leptodiaptomus angustilobius (100\%) & - & 1 \\
\hline 13 & Leptodiaptomus angustilobius $(86,2 \%)$ & & 4 \\
\hline 14 & Polyphemus pediculus $(72,2 \%)$ & & 2 \\
\hline 15 & - & - & 0 \\
\hline 16 & Chydorus sphaericus $(95,7 \%)$ & Alona affinis $(4,3 \%)$ & 2 \\
\hline 17 & Bosmina longirostris (80\%) & Graptoleberis testudinaria (20\%) & 2 \\
\hline 18 & Leptodiaptomus angustilobius (66,7\%) & Cyclops vicinus $(25 \%)$ & 4 \\
\hline 19 & Chydorus sphaericus $(93,8 \%)$ & - & 4 \\
\hline 20 & Leptodiaptomus angustilobius (88,5\%) & Chydorus sphaericus $(8,8 \%)$ & 3 \\
\hline 21 & Polyphemus pediculus $(61,2 \%)$ & Leptodiaptomus angustilobius $(24,3 \%)$ & 6 \\
\hline
\end{tabular}




\section{Acknowledgements}

The studies were supported by the Russian Science Foundation (Grant 14-14-00778). The material was collected by Chertoprud EM.

\section{References}

- Boxshall GA, Defaye D (2009) World checklist of freshwater Copepoda species. World Wide Web electronic publication. http://fada.biodiversity.be/group/show/19. Accession date: 2015710.

- Il'yna I, Lapshina E, Lavrenko N (1985) Rastitelnyi pokrov ZapadnoSibirskoi Ravniny. [The vegetation cover of the West Siberian Plain]. Nauka, Novosibirsk, 250 pp. [In Russian].

- Korovchinsky N (2004) Vetvistousie rakoobraznye otryada Ctenopoda mirovoy fauny (morfologia, sistematika, ecologia, zoogeografia). [Cladocerans of the order Ctenopoda of the world fauna (morphology, systematics, ecology, biogeography)]. KMK Press, Moscow, 410 pp. [In Russian].

- Kotov A, Forro L, Korovchinsky NM, Petrusek A (2013) World checklist of freshwater Cladocera species. World Wide Web electronic publication. http://fada.biodiversity.be/ group/show/17

- $\quad$ Nikiforov V (1997) The Gydan Peninsula Protected as Russia Creates Its 96th Strict Nature Reserve. World Wildlife Fund Arctic Bulletin 1: 15.

- Ongley E (1996) Chapter 13 - SEDIMENT MEASUREMENTS. In: Bartram J, Ballance R (Eds) Water Quality Monitoring - A Practical Guide to the Design and Implementation of Freshwater Quality Studies and Monitoring Programmes. CRC Press [ISBN 0419 223207 (Hbk) 0419217304 (Pbk)].

- $\quad$ Popov P (2012) Adaptaciya gidrobiontov k usloviyam obitaniya v vodoemah Subarctiki na primere ecologii ryb v vodoemah Subarktiki Zapadnoy Sibiri. Uchebnoe posobie. [Adaptation of hydrobionts to epy habitat conditions in the subarctic water bodies on the example of the fishes' ecology in the water bodies of the subarctic Western Siberia]. Novosibirsk, 255 pp. [In Russian].

- $\quad$ Semyonova L, Aleksuk V, SM D, TI L (2000) Vidovoe raznoobrazye zooplanktona vodoemov obskogo severa. Vestnik ecologii 1: 127-134. [In Russian].

- Sharapova T, Abdullina G (2004) K izucheniu vodnyh bespozvonochnyh uznyh tundr Zapadnoi Sibiri. Vestnik ecologii, lesovedenia i landshaftovedenia 5: 97-115. [In Russian]. 